\title{
Josephson Junctions with a synthetic antiferromagnetic interlayer
}

\author{
Mazin A. Khasawneh, W. P. Pratt, Jr., Norman O. Birge* \\ Department of Physics and Astronomy, Michigan State University, East Lansing, Michigan 48824-2320, USA
}

(Dated: November 3, 2018)

\begin{abstract}
We report measurements of the critical current vs. Co thickness in $\mathrm{Nb} / \mathrm{Cu} / \mathrm{Co} / \mathrm{Ru} / \mathrm{Co} / \mathrm{Cu} / \mathrm{Nb}$ Josephson junctions, where the inner $\mathrm{Co} / \mathrm{Ru} / \mathrm{Co}$ trilayer is a "synthetic antiferromagnet" with the magnetizations of the two Co layers coupled antiparallel to each other via the $0.6 \mathrm{~nm}$-thick $\mathrm{Ru}$ layer. Due to the antiparallel magnetization alignment, the net intrinsic magnetic flux in the junction is nearly zero, and such junctions exhibit excellent Fraunhofer patterns in the critical current vs. applied magnetic field, even with total Co thicknesses as large as $23 \mathrm{~nm}$. There are no apparent oscillations in the critical current vs. Co thickness, consistent with theoretical expectations for this situation. The critical current of the junctions decays over 4 orders of magnitude as the total Co thickness increases from 3 to $23 \mathrm{~nm}$. These junctions may serve as useful templates for future explorations of spin-triplet superconducting correlations, which are predicted to occur in superconducting/ferromagnetic hybrid systems in the presence of certain types of magnetic inhomogeneity.
\end{abstract}

PACS numbers: 74.50.+r, 74.45.+c, 75.70.Cn

Superconducting/ferromagnetic (S/F) hybrid systems have received much attention in the past decade. $\frac{1}{\underline{H}}$ When a conventional spin-singlet Cooper pair crosses the $\mathrm{S} / \mathrm{F}$ interface, the two electrons enter different spin bands, hence the pair picks up a momentum shift proportional to the exchange energy $\underline{2}$ This physical process leads to a number of oscillatory phenomena in S/F systems, including oscillations in the $T_{c}$ of $\mathrm{S} / \mathrm{F}$ bilayers and in the critical current of $\mathrm{S} / \mathrm{F} / \mathrm{S}$ Josephson junctions as a function of F-layer thickness. $\stackrel{1}{*}$ There are proposals to use $\mathrm{S} / \mathrm{F} / \mathrm{S}$ $\pi$-junctions as components in superconducting circuits or in various quantum computing schemes.

A relatively recent development is the prediction of a new kind of spin-triplet pair correlations in S/F induced in conventional S/F systems by the presence of certain forms of magnetic inhomogeneity ${ }^{3.4}$ Unlike spin-singlet pairs, spin-triplet pairs are not subject to the exchange field, hence they should propagate long distances in a ferromagnetic material - limited only by the temperature or by spin-flip or spin-orbit scattering. One place to search for spin-triplet correlations is in thick S/F/S Josephson junctions, where the spin-singlet supercurrent is exponentially suppressed by the exchange field $\stackrel{\underline{\underline{5}}}{\mathrm{~T}}$ Depending on their geometry and the type of $\mathrm{F}$ material, however, thick $\mathrm{S} / \mathrm{F} / \mathrm{S}$ junctions may contain a large amount of intrinsic magnetic flux, which distorts the "Fraunhofer" pattern of the critical current vs. applied magnetic field and reduces the reliability of critical current measurements.

We report here measurements of $\mathrm{Nb} / \mathrm{Cu} / \mathrm{Co} / \mathrm{Ru} / \mathrm{Co} / \mathrm{Cu} / \mathrm{Nb}$ Josephson junctions, where the central $\mathrm{Co} / \mathrm{Ru} / \mathrm{Co}$ trilayer is a "synthetic antiferromagnet" with the magnetizations of the two Co layers exchange-coupled antiparallel to each other via the 0.6 nm-thick Ru layer. ${ }^{6}$ The total Co thickness was varied between 3 and $23 \mathrm{~nm}$ - much thicker than in previous studies of $\mathrm{S} / \mathrm{F} / \mathrm{S}$ junctions using Co with thicknesses up

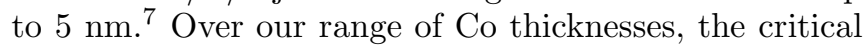
current drops by more than 4 orders of magnitude, while the critical current vs. applied magnetic field exhibits a nearly perfect Fraunhofer pattern over the entire range. We do not observe any signature of spin-triplet correlations in these samples, but we suggest that they may serve as a useful platform for future searches for triplet correlations, perhaps by adding additional magnetic layers with inhomogeneous magnetization adjacent to the Nb layers.

Multilayer samples of the form $\mathrm{Nb}(150) / \mathrm{Cu}(5) / \mathrm{Co}(\mathrm{x}) / \mathrm{Ru}(0.6) / \mathrm{Co}(\mathrm{x}) / \mathrm{Cu}(5) / \mathrm{Nb}(25) / \mathrm{Au}(15)$, with all thicknesses in nm, were grown by dc triode sputtering in an Ar pressure of 2.5 mTorr, in a system with base pressure of $2 \times 10^{-8}$ Torr. The thin $\mathrm{Cu}$ layers appear to change the growth characteristics of the Co layers, and result in larger critical current of the junctions for thick Co layers. (Results for samples with and without the $\mathrm{Cu}$ layers will be shown below.) The total Co thickness, $d_{C o}=2 x$, was varied between 3 and $23 \mathrm{~nm}$. The multilayers were patterned into circular pillars of diameters 10, 20, 40, and $80 \mu \mathrm{m}$ using an image reversal photolithographic process and Ar ion milling. The milling was followed immediately by deposition of $160 \mathrm{~nm}$ of $\mathrm{SiO}_{x}$, then lift-off of the photoresist mask. Finally, top Nb electrodes of thickness $200 \mathrm{~nm}$ were deposited by sputtering. A schematic diagram of the sample geometry is shown in Fig. 1. All critical current measurements were performed at $4.2 \mathrm{~K}$ with the samples inside a Cryoperm magnetic shield, using a SQUID-based current comparator method $\stackrel{8}{-}$ Current-voltage characteristics of all samples followed the standard form for overdamped Josephson junctions.

A valuable tool for characterizing the quality of Josephson junctions is the measurement of critical current vs. magnetic field applied perpendicular to the current direction. Observation of a good Fraunhofer pattern for junctions guarantees that the current flow is uniform across the junction, and that there are no shorts in the surrounding insulator. Observation of the Fraunhofer pattern in $\mathrm{S} / \mathrm{F} / \mathrm{S}$ junctions with strong ferromagnets, 


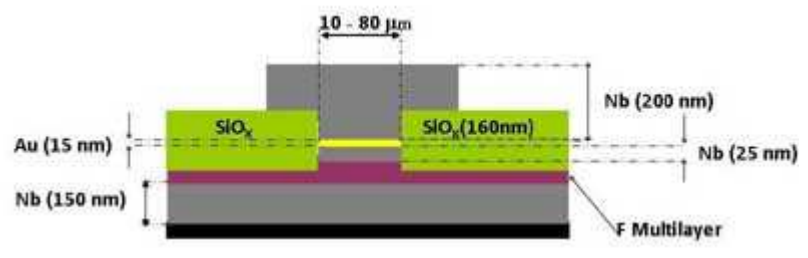

FIG. 1: (color online). Schematic diagram of S/F/S Josephson junction cross section, where the " $F$ multilayer" refers to the $\mathrm{Co} / \mathrm{Ru} / \mathrm{Co}$ trilayer, with or without additional $\mathrm{Cu}$ buffer layers adjacent to the $\mathrm{Nb}$ electrodes. Current flow is in the vertical direction. The magnetic field is applied in the plane of the layers, i.e. perpendicular to the current direction.

however, can be problematic, due to the intrinsic magnetic flux of the ferromagnetic domains. For sufficiently thin F layers, the Fraunhofer patterns can be extremely good $\stackrel{9}{9}$ In junctions with extremely small lateral dimensions, good Fraunhofer patterns can be obtained over a larger range of F-layer thickness $\underline{\underline{10}}$ But for sufficiently thick F layers, the Fraunhofer pattern becomes random, with no clear central maximum. An example for a circular junction of diameter $40 \mu \mathrm{m}$, with a single Co layer 5 nm thick, is shown in Fig. 2. The deep minima in $I_{c}$ at $H=-5$ and +8 Oe demonstrate that there are no shorts in the oxide surrounding the junction. The overall pattern, however, is quite random, due to the randomness of the magnetic domain structure of the Co film. Similar random Fraunhofer patterns have been seen previously in $\mathrm{S} / \mathrm{F} / \mathrm{S}$ junctions containing other strong ferromagnetic materials: $\mathrm{Gd}^{11}$ and $\mathrm{Ni}^{12}$

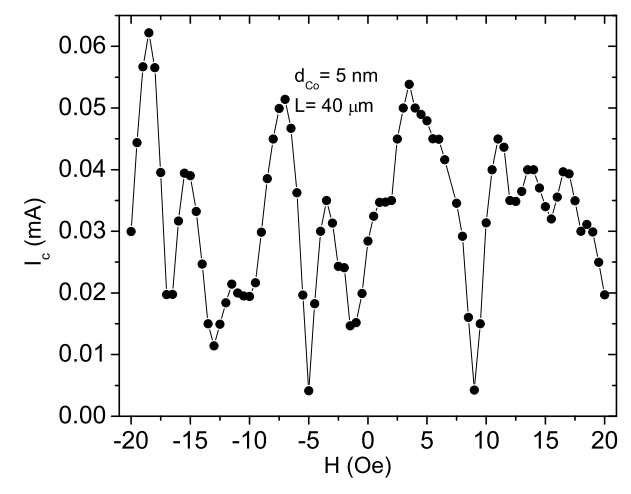

FIG. 2: Critical current vs. magnetic field applied in the film plane (perpendicular to the current direction) for a $\mathrm{Nb} / \mathrm{Co} / \mathrm{Nb}$ circular Josephson junction of diameter $40 \mu \mathrm{m}$ and $d_{C o}=5 \mathrm{~nm}$.

Fabrication of Josephson junctions containing the synthetic antiferromagnetic trilayer, $\mathrm{Co}(\mathrm{x}) / \mathrm{Ru}(0.6) / \mathrm{Co}(\mathrm{x})$, circumvents this problem. Fig. [3] shows Fraunhofer patterns for four samples with total Co thicknesses varying

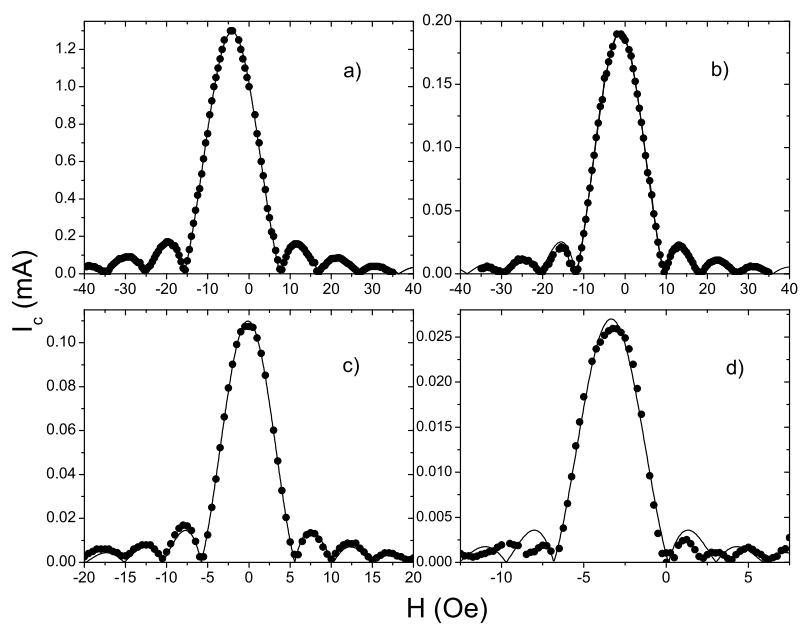

FIG. 3: Critical current vs. applied magnetic field obtained for $\mathrm{Nb} / \mathrm{Cu} / \mathrm{Co} / \mathrm{Ru} / \mathrm{Co} / \mathrm{Cu} / \mathrm{Nb}$ circular Josephson junctions with different total thickness of the Co layers: a) $6.1 \mathrm{~nm}, \mathrm{~b}$ ) $11 \mathrm{~nm}$, c) $18 \mathrm{~nm}$, and d) $23 \mathrm{~nm}$. The pillar diameters $w$ are 10, 10, 20, and $40 \mu \mathrm{m}$, respectively. The solid lines are fits to Eq. (1).

from 6.1 to $23 \mathrm{~nm}$. The first three patterns are nearly perfect, while the last one is still extremely good. The maximum field shift of the patterns is a few Oe, which indicates a very strong antiferromagnetic coupling between the top and bottom Co layers. Solid lines are fits to the theoretical Airy formula for junctions with circular cross section:

$$
I_{c}(\Phi)=I_{c}(0) \frac{2 \times J_{1}\left(\frac{\pi \Phi}{\Phi_{0}}\right)}{\left(\frac{\pi \Phi}{\Phi_{0}}\right)},
$$

where $J_{1}$ is the Bessel function of the first kind, $\Phi_{0}=$ $h / 2 e$ is the flux quantum, $\Phi=H_{e x t}\left(2 \lambda_{L}+d\right) w$ is the magnetic flux penetrating the junction with $\lambda_{L}$ the London penetration depth, $w$ the junction diameter and $d$ the barrier thickness. The antiferromagnet coupling of the $\mathrm{Co} / \mathrm{Ru} / \mathrm{Co}$ trilayer was confirmed independently from measurements of magnetization $(M)$ vs. applied field for $\mathrm{Co}(4) / \mathrm{Ru} / \mathrm{Co}(4)$ trilayers with varying thicknesses of $\mathrm{Ru}$. The coupling was evident for Ru thicknesses of 0.6 , $0.7,0.8$, and $0.9 \mathrm{~nm}$. Fig. 4 4 shows $M$ vs. $H$ for a sample with $\mathrm{Ru}$ thickness of $0.6 \mathrm{~nm}$. $M$ doesn't saturate until $H$ is at least $5 \mathrm{kOe}$, and there is very little hysteresis between curves with $H$ increasing and decreasing.

We subjected one Josephson junction sample to a series of large in-plane magnetic fields, then re-measured $I_{c}$ vs. $H$ at low field. The resulting Fraunhofer patterns showed only slight distortion after applying fields as large as $5 \mathrm{kOe}$. After applying $10 \mathrm{kOe}$ the central peak in the Fraunhofer pattern split into two peaks of about half the original magnitude. After warming the sample to room temperature and cooling back to $4.2 \mathrm{~K}$, an excellent Fraunhofer pattern was obtained once again.

The dependence of $I_{c}$ on total Co thickness $\left(d_{C o}\right)$ is 


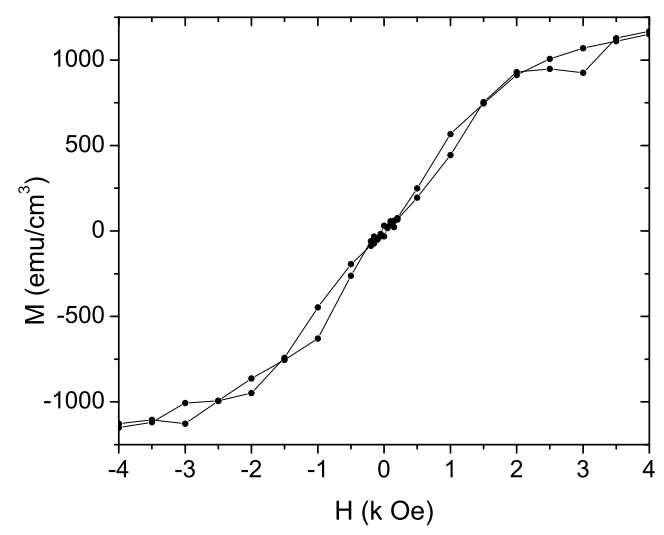

FIG. 4: Magnetization vs. applied field at $T=10 \mathrm{~K}$ for a $\mathrm{Co}(4) / \mathrm{Ru}(0.6) / \mathrm{Co}(4)$ trilayer grown on $150 \mathrm{~nm}$ of $\mathrm{Nb}$.

summarized in Fig. 5] The figure shows two sets of data: black circles represent the samples fabricated with the $\mathrm{Cu}$ buffer layer, while red triangles represent samples fabricated earlier without the $\mathrm{Cu}$ layer. In both cases $I_{c}$ decays exponentially with $d_{C o}$. In samples without the $\mathrm{Cu}$, the decay is faster than in samples with $\mathrm{Cu}$. We focus first on the larger data set - the samples with $\mathrm{Cu}$. An immediate question is whether these samples can be $\pi$-junctions; i.e. does $I_{c}$ oscillate with $d_{C o}$ ? While the data do not display convincing oscillations, there are a few data points (e.g. for $d_{C o}=4.0,18$, and $23 \mathrm{~nm}$ ) that are substantially above or below their neighbors. To address the question of oscillations, we fabricated a set of samples in one sputtering run with closely spaced Co layer thicknesses in the range $4.3-6.1 \mathrm{~nm}$. Those samples do not exhibit any local minima in $I_{c}$, whereas Robinson et al $\underline{\underline{7}}$ observed a spacing of $1.0 \mathrm{~nm}$ between local minima for $\mathrm{Nb} / \mathrm{Co} / \mathrm{Nb}$ junctions containing a single Co layer.

Several theoretical papers address the expected behavior of $I_{c}$ vs. $d_{C o}$ for Josephson junctions containing two magnetic layers with non-collinear magnetizations $\frac{13,14,15}{1}$ We discuss only the situation relevant to our experiments, where the two ferromagnetic layers have equal thickness and antiparallel magnetizations. In the ballistic limit, such $\mathrm{S} / \mathrm{F} / \mathrm{F} / \mathrm{S}$ junctions are predicted to behave similarly to $\mathrm{S} / \mathrm{N} / \mathrm{S}$ junctions - with a slow algebraic decay and no oscillations in $I_{c}$ - because the relative phase shift acquired by the two electrons of a Cooper pair as they travel through the first $\mathrm{F}$ layer is exactly cancelled by the phase shift they acquire through the second $\mathrm{F}$ layer $\stackrel{13}{ }$ In the presence of disorder the critical current decays exponentially with $\mathrm{F}$ layer thickness, but still without any oscillations of the kind associated with $\mathrm{S} / \mathrm{F} / \mathrm{S}$ junctions. Our data are consistent with this picture.

To extract quantitative information from our data, we must go a step further with the theory. The theoretical works cited above calculate the exact form of the $I_{c}$ decay only in certain limits, e.g. for the pure ballistic case

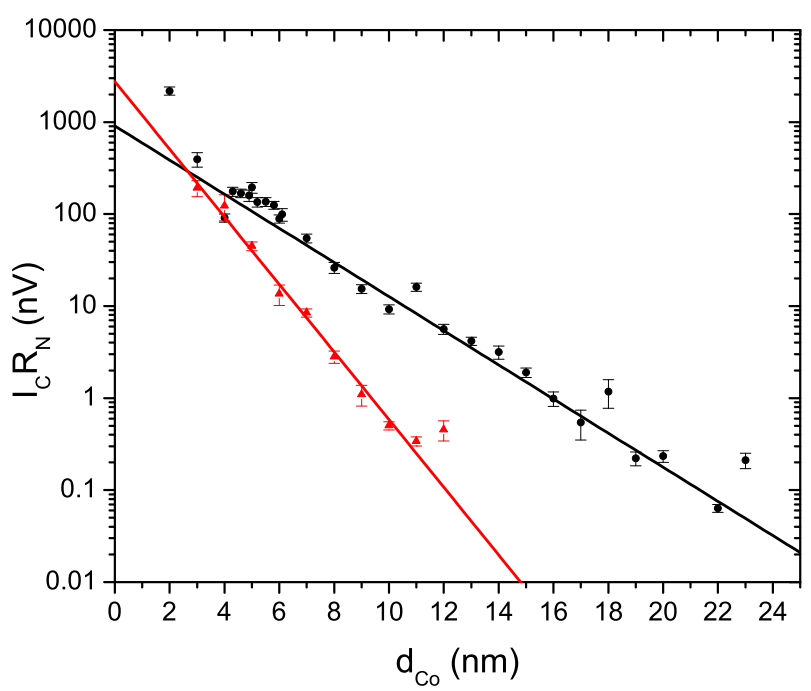

FIG. 5: (color online). Product of critical current times normal state resistance vs. total Co thickness for all of our SAF Josephson junctions. Red points (triangles) are data for samples without $\mathrm{Cu}$ buffer layers, while black points (circles) are data for samples with $\mathrm{Cu}$ buffer layers. Error bars represent the standard deviation of measurements taken on more than one pillar on the same substrate, with the minimum uncertainty chosen to be $10 \%$. The solid lines are fits to a simple exponential decay, with decay lengths of $1.18 \pm 0.05 \mathrm{~nm}$ and $2.34 \pm 0.08 \mathrm{~nm}$, respectively.

with no elastic scattering and for the diffusive limit with $E_{\text {ex }} \tau \ll \hbar$, where $\tau$ is the mean free time between collisions. Josephson junctions with Co, however, fall into an intermediate limit, where $E_{e x} \tau>\hbar$, but $\Delta \tau \ll \hbar$, with $\Delta$ the superconducting gap. Although theories in the intermediate limit do not exist for $\mathrm{S} / \mathrm{F} / \mathrm{F} / \mathrm{S}$ junctions, they do exist for $\mathrm{S} / \mathrm{F} / \mathrm{S}$ junctions, $\stackrel{17,18}{1}$ and predict exponential decay of $I_{c}$ with a decay constant equal to the mean free path in the $\mathrm{F}$ material. (Theories for $\mathrm{S} / \mathrm{F} / \mathrm{S}$ junctions also predict oscillations, which are not present in the $\mathrm{S} / \mathrm{F} / \mathrm{F} / \mathrm{S}$ case studied here.) The solid lines in Fig. 5 are least-squares fits of an exponential decay to our two data sets, with decay lengths $2.34 \pm 0.08 \mathrm{~nm}$ for the samples with $\mathrm{Cu}$ buffer layers and $1.18 \pm 0.05 \mathrm{~nm}$ for the samples without $\mathrm{Cu}$. This analysis suggests that the mean free path in the Co grown on the $\mathrm{Cu}$ buffer layer is longer than in the Co grown directly on $\mathrm{Nb}$, probably due to less strain in the former case. Confirmation of that hypothesis would require analysis of the grain structure of our films by transmission electron microscopy.

It is instructive to compare our results with those of Robinson et al $\stackrel{7,10}{,}$, who studied $\mathrm{S} / \mathrm{F} / \mathrm{S}$ junctions made with the strong ferromagnets $\mathrm{Co}, \mathrm{Ni}, \mathrm{Fe}$, and $\mathrm{Py}$, all of which are believed to lie in the intermediate limit defined above. Those workers found that, for $\mathrm{Ni}$ and $\mathrm{Py}$, the $I_{c}$ vs. $d_{F}$ data followed an algebraic decay for small $d_{F}$ and an exponential decay for larger $d_{F}$, with the crossover interpreted as occurring when $d_{F}$ surpasses the mean free 
path, $l_{e}$. For Co, the data could be fit with either an algebraic or exponential decay over the thickness range studied, $0.8-5 \mathrm{~nm}$. As shown in Fig. 5, our $I_{c}$ data decay exponentially over the entire range of $d_{C o}=2-23 \mathrm{~nm}$, with the possible exception of our first data point. Given the extra scattering in our samples from the two $\mathrm{Co} / \mathrm{Ru}$ interfaces,$\frac{16}{r}$ it is not surprising that $l_{e}<d_{C o}$ over the entire range of Co thicknesses we measured. What is more surprising is that, if we were to plot the data of Robinson et al $\stackrel{10}{\underline{10}}$ (ignoring the oscillations) in Fig. [5. they would lie a factor of 100 higher than our data over the narrow thickness range covered by both experiments. This suggests that the thin Ru layer severely suppresses $I_{c}$, possibly due to spin memory loss at the $\mathrm{Co} / \mathrm{Ru}$ interfaces.

The single exponential decay of $I_{c}$ vs. $d_{C o}$ shown in Fig. 5 indicates a lack of spin-triplet superconducting correlations in these samples, which would manifest themselves as a crossover to a slower decay with increasing $d_{C o}$. (An optimist might consider the point at $d_{C o}=23 \mathrm{~nm}$ as a hopeful sign, but a sample with $d_{C o}=24 \mathrm{~nm}$ exhibited a very small supercurrent and no Fraunhofer pattern, hence it was excluded from the Figure.) There are several possible reasons why we do not observe the long-range triplet correlations (LRTC). First, there could be substantial spin memory loss at the $\mathrm{Co} / \mathrm{Ru}$ interfaces - an issue we intend to clarify in the near future for our samples using giant magnetoresistance techniques. Second, the amplitude of the LRTC generated at the S/F interfaces may be too small to measure. This could occur either if the domain structure in the Co films contains mostly domains aligned along a single directions in space (the LRTC requires non-collinear magnetizations), or if the LRTC component has random phases at adjacent Co domain walls, and hence averages to zero over the lateral dimensions of the samples $\frac{19}{19}$ The latter situation could be ameliorated by fabricating samples with smaller lateral dimensions, while both issues could be addressed by utilizing a magnetic material with a well-characterized form of magnetic inhomogeneity, such as the spiral magnetic structure occurring in materials such as Ho, 20,21,22

In this context, we note that optimizing the generation of the LRTC at the S/F interface may involve a choice of materials that does not optimize propagation of the LRTC through the subsequent ferromagnetic materials. It is here where we believe the Josephson junctions reported in this paper may hold the most promise. One can imagine producing samples of the form $\mathrm{S} / \mathrm{X} / \mathrm{SAF} / \mathrm{X} / \mathrm{S}$, where $\mathrm{X}$ is a magnetic material chosen to optimize LRTC generation, while SAF is a suitable synthetic antiferromagnet with little spin memory loss, either the $\mathrm{Co} / \mathrm{Ru} / \mathrm{Co}$ trilayer studied here or a weaker $\mathrm{SAF}$ such as $\mathrm{Co} / \mathrm{Cu} / \mathrm{Co} \stackrel{23}{2}$ Once the SAF layer becomes sufficiently thick (greater than about $23 \mathrm{~nm}$ for the case of the Co SAF studied here), the singlet supercurrent is suppressed by over 4 orders of magnitude. Generation of the LRTC at the S/X interfaces would then be manifested as a long-range spin-triplet supercurrent that persists out to SAF thicknesses far beyond what has been measured here.

We are grateful to S. Bergeret, Y. Blanter, D. van Harlingen, A. Volkov, and K. Efetov for helpful discussions, and to $\mathrm{B}$. Bi, T. Khaire, R. Loloee, and Y. Wang for technical assistance. This work was supported by the Department of Energy under grant DE-FG02-06ER46341.
* Electronic address: birge@pa.msu.edu

1 A.I. Buzdin, Rev. Mod. Phys. 77, 935 (2005).

2 E.A. Demler, G.B. Arnold, and M.R. Beasley, Phys. Rev. B 55, 15174 (1997).

3 F.S. Bergeret, A.F. Volkov, and K.B. Efetov, Phys. Rev. Lett. 86, 4096 (2001).

4 A. Kadigrobov, R.I. Shekhter, and M. Jonson, Europhys. Lett. 54(3), 394 (2001).

${ }^{5}$ R.S. Keizer, S.T.B. Goennenwein, T.M. Klapwijk, G. Xiao, and A. Gupta, Nature (London) 439, 825 (2006).

6 S.S.P. Parkin, N. More, and K.P. Roche, Phys. Rev. Lett. 64, 2304 (1990).

7 J.W.A. Robinson, S. Piano, G. Burnell, C. Bell and M.G. Blamire, Phys. Rev. Lett. 97, 177003 (2006).

8 D. Edmunds, W. Pratt, and J. Rowlands, Rev. of Sci. Instr. 51, 1516 (1980).

9 D. Sprungmann, K. Westerholt, H. Zabel, M. Weides, and H. Kohlstedt, J. Phys. D: Appl. Phys. 42, 075005 (2009).

10 J.W.A. Robinson, S. Piano, G. Burnell, C. Bell and M. G. Blamire, Phys. Rev. B 76, 094522 (2007).

11 O. Bourgeois, P. Gandit, J. Lesueur, A. Sulpice, X. Grison, and J. Chaussy, Eur. Phys. J. B 21, 75 (2001).

12 T. Khaire, W.P. Pratt, Jr., and N.O. Birge, Phys. Rev. B
79, 094523 (2009).

13 Ya. M. Blanter and F.W.J. Hekking, Phys. Rev. B 69, 024525 (2004).

14 A. Vedyayev, C. Lacroix, N. Pugach and N. Ryzhanova, Europhys. Lett. 71, 679 (2005).

15 B. Crouzy, S. Tollis, and D.A. Ivanov, Phys. Rev. B 75, 054503 (2007).

16 C. Ahn, K.-H. Shin, and W.P. Pratt, Jr., Appl. Phys. Lett. 92, 102509 (2008).

17 F.S. Bergeret, A.F. Volkov, and K.B. Efetov, Phys. Rev. B 64, 134506 (2001).

18 O. Kashuba, Y.M. Blanter, V.I. Falko, Phys. Rev. B 75, 132502 (2007).

19 S. Bergeret, private communication.

20 I. Sosnin, H. Cho, V.T. Petrashov, and A.F. Volkov, Phys. Rev. Lett. 96, 157002 (2006).

21 A.F. Volkov, A. Anishchanka, and K.B. Efetov, Phys. Rev. B 73, 104412 (2006).

22 G.B. Halasz, J.W.A. Robinson, M.G. Blamire, and J.F. Annett, arXiv:0901.2024

23 S.S.P. Parkin, R. Bhadra, and K.P. Roche, Phys. Rev. Lett. 66, 2152 (1991). 